\title{
Sliding Window Technique to Detect the Presence of LTE
}

\author{
Rakibul Hasan *, Mohammad Saquib * and Jeffrey Boksiner* \\ \# The University of Texas at Dallas, Richardson, TX, 75080 \\ *US Army RDECOM CERDEC S\&TCD, Aberdeen Proving Ground, MD \\ \# \{mxh079000, saquib $\} @$ utdallas.edu
}

\begin{abstract}
This paper describes the synchronization signals used by the Long Term Evolution (LTE) with a main focus on the detection of the presence of LTE coverage. The LTE system uses its central 62 subcarriers for the purpose of synchronization and broadcast system information. We use sliding window technique over the fading channels with different number of channel taps to detect the presence of two synchronization signals which in turns indicates the presence of LTE.
\end{abstract}

\section{INTRODUCTION}

The 3rd Generation Partnership Project (3GPP) LTE system achieves higher user throughput and frequency efficiency than its older systems. In the downlink, Orthogonal Frequency Division Multiplexing (OFDM) based radio access was adopted and single-carrier frequency-division multiple access is used in the uplink. LTE uses frequency division duplex (FDD) or time division duplex (TDD)[1]. At the beginning of communication, the user equipment (UE) must perform a cell search. Two types of synchronization signals, primary synchronization signal (PSS) and secondary synchronization signal (SSS) are used for initial slot and frame synchronization[2]. Synchronization procedure is necessary to determine the time and frequency parameters, to demodulate the downlink signals and to acquire certain critical system parameters, e.g. cyclic prefix length.

In our work, we focus on detecting the presence of these synchronization sequences for the purpose of Dynamic Spectrum Access (DSA) without any prior knowledge of the signal over multi-tap channel. Since the secondary user (SU) can start scanning at any instant of an LTE frame, we tend to capture as much energy as possible by employing three different sliding window techniques.

\section{SyNCHRONIZATION SignALS}

The synchronization signals are transmitted periodically, twice per $10 \mathrm{~ms}$ radio frame[3]. In the frequency domain, both PSS and SSS are always transmitted in the central six Resource Blocks (RBs) and allows the UE to synchronize to the network without any a priori knowledge of the allocated bandwidth[5]. This means that the five resource elements at each extremity of each synchronization sequence are left unused.

\section{A. Primary Synchronization Signal (PSS)}

In an FDD cell, the PSS is always located in the last OFDM symbol of the 1st and 11th slots of each radio frame as shown in Fig 1 for normal Cyclic Prefix (CP) length. The PSS uses sequences known as Zadoff-Chu (ZC) sequence. The ZC sequence of odd-length $N_{Z C}$ is described at page $155 \mathrm{in}[2]$ as

$$
Z_{q}(n)=\exp \left[-j 2 \pi q \frac{\frac{n(n+1)}{2}+l n}{N_{Z C}}\right],
$$

where $q \epsilon\left\{1 \ldots N_{Z C}-1\right\}$ is the ZC sequence root index and $n=0,1 \ldots N_{Z C}-1$, is any integer. In LTE, $q=25,29,34$ and $l=0$ are used for simplicity. The PSS is constructed from a frequency-domain ZC sequence of length 63, with the middle element punctured to avoid transmitting on the d.c. subcarrier.

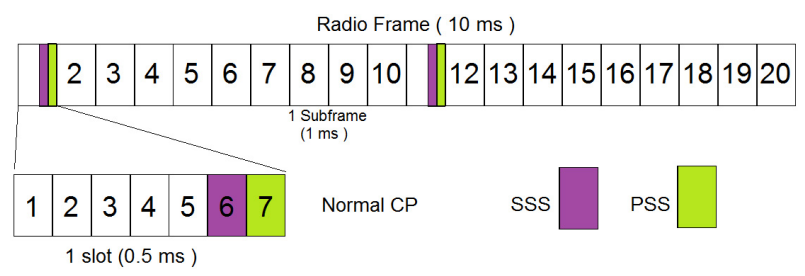

Fig. 1. Structure of PSS and SSS in time domain.

\section{B. Secondary Synchronization Signal (SSS)}

SSS is located just before PSS which enables the UE to acquires the slot boundary timing independently of the CP length. SSS is generated from the combination of two length31 sequences defined as

$$
\begin{aligned}
d(2 n) & = \begin{cases}s_{0}^{\left(m_{0}\right)}(n) c_{0}(n) ; & \text { subframe 0, } \\
s_{1}^{\left(m_{1}\right)}(n) c_{0}(n) ; & \text { subframe 5, }\end{cases} \\
d(2 n+1) & = \begin{cases}s_{1}^{\left(m_{1}\right)}(n) c_{1}(n) z_{1}^{\left(m_{0}\right)}(n) ; & \text { subframe 0, } \\
s_{0}^{\left(m_{0}\right)}(n) c_{1}(n) z_{1}^{\left(m_{1}\right)}(n) ; & \text { subframe 5, }\end{cases}
\end{aligned}
$$

where $s(n), c(n)$ and $z(n)$ are all scrambling sequences. The indices $m_{0}$ and $m_{1}$ are derived from PSS and detail expressions can be found in [6].

\section{SySTEM MODEL}

Since initially the UE has no prior information, we propose a modified energy based detector where sliding window technique is applied to estimate final decision statistics variable. In our system model, we assume that the LTE signal 
is propagating through multi-path fading channel and SU is scanning continuously for half LTE frame $(5 \mathrm{~ms})$ in time domain and central six resource blocks in frequency domain. Following steps are involved to execute the proposed sliding window technique.

- Step 1: SU breaks down the received signal by applying a rectangular window of length two OFDM symbols period $(142.8 \mu \mathrm{s})$ in time domain.

- Step 2: SU then removes the CP and applies the energy based decision formula $Z_{\mathrm{ed}}^{(m)}=\sum_{i=1}^{N_{w}}\left|r_{i}^{(m)}\right|^{2}$ to measure the power of the $m \underline{\text { th }}$ window. Here $N_{w}$ is the length of the rectangular window and $r_{i}^{(m)}$ is the received signal at $m^{\text {th }}$ window.

- Step 3: Once the window number reaches the total number of windows $N_{t}$, appropriate weighting factor is multiplied with decision variable $Z_{\mathrm{ed}}^{(m)}$ and $Z_{\mathrm{ed}}^{(m)}$ from all the windows are then added together and compare with a predefined threshold to determine the presence of LTE coverage.

\section{ANALYSIS}

Most single threshold signal detection problem is expressed as two hypotheses $H_{0}$ and $H_{1}$, which represent the absence and presence of the primary signal respectively. When the signal is absent (i.e. case $H_{0}$ ), only complex additive white Gaussian noise (AWGN) is present within every window and final decision statistics $\Gamma$ can be expressed as

$$
\Gamma=\sum_{m=1}^{N_{t}} w^{(m)} Z_{\mathrm{ed}}^{(m)}=\sum_{m=1}^{N_{t}} \sum_{v=1}^{N_{w}} w^{(m)}\left|n_{v}^{(m)}\right|^{2},
$$

where $n_{v}^{(m)}$ is the $v^{\text {th }}$ noise sample of $m^{\text {th }}$ window and $w^{(m)}$ is the real valued weighting factor of the $m^{\text {th }}$ window. Performance of the proposed system will vary based on the different arrangements of the weighting factor. We consider following three different cases.

1) Case 1: When $w^{(m)}=1$ for all the windows, the expression in (1) will act as summation of all the elements in different windows. If we rearrange this summation such that the correlated terms from every window are added together to form an individual component $\Gamma_{l}$, then the above summation can be rearranged as

$$
\Gamma=\sum_{l=1}^{N} \Gamma_{l}
$$

where $N$ is the total number of samples and $\Gamma_{l}$ is expressed as

$$
\begin{cases}l\left|n_{l}\right|^{2}, & 1 \leq l<N_{w} \\ N_{w}\left|n_{l}\right|^{2}, & N_{w} \leq l \leq\left(N-N_{w}+1\right) \\ (N-l+1)\left|n_{l}\right|^{2}, & \left(N-N_{w}+1\right)<l \leq N\end{cases}
$$

Due to this reshuffle, $\Gamma_{l}$ are independent of one another so that mean and variance of $\Gamma$ for $H_{0}$ can be expressed as

$$
\mathbb{E}\left[\Gamma \mid H_{0}\right]=\sum_{l=1}^{N} \mu_{\Gamma_{l}} ; \quad \operatorname{var}\left[\Gamma \mid H_{0}\right]=\sum_{l=1}^{N} \sigma_{\Gamma_{l}}^{2}
$$

Every $n_{l}$ from (3) is zero mean with variance $\sigma_{n}^{2}$ while mean $\mu_{\Gamma_{l}}$ in (4) can be formulated as

$$
\begin{cases}l \sigma_{n}^{2}, & 1 \leq l<N_{w}, \\ N_{w} \sigma_{n}^{2}, & N_{w} \leq l \leq\left(N-N_{w}+1\right), \\ (N-l+1) \sigma_{n}^{2}, & \left(N-N_{w}+1\right)<l \leq N .\end{cases}
$$

Variance $\sigma_{\Gamma_{l}}^{2}$ in (4) can also be expressed as

$$
\begin{cases}l^{2} \mathbb{E}\left[\left|n_{l}\right|^{4}\right]-\left(\mu_{\Gamma_{l}}\right)^{2}, & 1 \leq l<N_{w}, \\ N_{w}^{2} \mathbb{E}\left[\left|n_{l}\right|^{4}\right]-\left(\mu_{\Gamma_{l}}\right)^{2}, & N_{w} \leq l \leq\left(N-N_{w}+1\right), \\ (N-l+1)^{2} \mathbb{E}\left[\left|n_{l}\right|^{4}\right]-\left(\mu_{\Gamma_{l}}\right)^{2}, & \left(N-N_{w}+1\right)<l \leq N,\end{cases}
$$

where $\mathbb{E}\left[\left|n_{l}\right|^{4}\right]=2 \sigma_{n}^{4}$. Probability of false alarm $P_{f}$ for a threshold value $V_{T}$ can be expressed as

$$
P_{f}=P\left[\Gamma>V_{T} \mid H_{0}\right]=P\left[\frac{\Gamma-\mu_{\Gamma}}{\sigma_{\Gamma}}>\frac{V_{T}-\mu_{\Gamma}}{\sigma_{\Gamma}} \mid H_{0}\right] .
$$

According to the application of the Central Limit Theorem, $\frac{\Gamma-\mu_{\Gamma}}{\sigma_{\Gamma}}$ becomes normal distribution with mean zero and variance one. We can derive a closed form expression of the threshold in the following manner:

$$
\begin{aligned}
\left(1-P_{f}\right) & =\frac{1}{2}\left[1+\operatorname{erf}\left(\frac{V_{T}-\mu_{\Gamma}}{\sqrt{2} \sigma_{\Gamma}}\right)\right] \longrightarrow \\
V_{T} & =\operatorname{erf}^{-1}\left(1-2 P_{f}\right) \sqrt{2} \sigma_{\Gamma}+\mu_{\Gamma} .
\end{aligned}
$$

where erf represents error function.

2) Case 2: When weighting factor $w^{(m)}$ is expressed as

$$
w^{(m)}= \begin{cases}1, & m^{\text {th }} \text { window has maximum value } \\ 0, & \text { otherwise. }\end{cases}
$$

In other words, we are only considering maximum $Z_{\text {ed }}^{(m)}$ among $Z_{\text {ed }}^{(m)}$ from all the windows and compare it with a threshold to calculate both $P_{f}$ and $P_{d}$ (i.e. probability of detection). Since the joint probability distribution of correlated random variables generated from different windows is unknown, it is difficult to find a closed form expression of $V_{T}$ for this case. Thus we evaluate the performance of our system numerically.

3) Case 3: When weighting factor $w^{(m)}$ is expressed as

$$
w^{(a)}= \begin{cases}\exp \left(-\frac{a-1}{k}\right), & \text { top } k \text { maximum values, } \\ 0, & \text { otherwise, }\end{cases}
$$

where $a=1,2, \ldots k$ and $k$ is any positive integer. In this case, instead of considering only one window (i.e. case 2), we are considering those $k$ windows having top $k$ maximum values of $Z_{\mathrm{ed}}^{(m)}$ and multiply them with the above weighting factors. Similar to case 2, we are also evaluating all the performance matrices from simulation. 


\section{NUMERICAL RESUlTS}

We evaluated the performance of the proposed algorithm for Rayleigh fading channels with Monte Carlo simulation. The performance of our system has been probed through MATLAB simulation using one LTE frame with normal CP. Throughout our simulation, we assume that SU can search for synchronization signal at any instant of an LTE frame while the channel remains unchanged during a single LTE frame. Note that we use root value $q=25$ to generate $\mathrm{ZC}$ sequence and decision statistics are collected and averaged over 10000 trials.

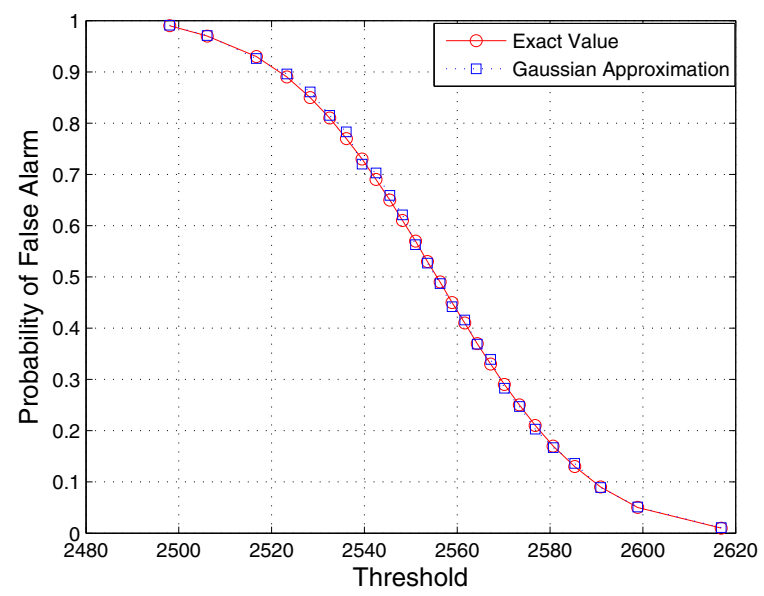

Fig. 2. Threshold vs. Probability of false alarm at $\mathrm{SNR}=0 \mathrm{~dB}$ for case 1 .

In Fig. 2, we have demonstrated the change of threshold with respect to the change of $P_{f}$ for signal to noise ratio $(\mathrm{SNR})=0 \mathrm{~dB}$ for case 1 . We first calculate $V_{T}$ using equation (7) and then use that threshold to evaluate $P_{f}$. It can be noticed that theoretical and simulation results closely matches.

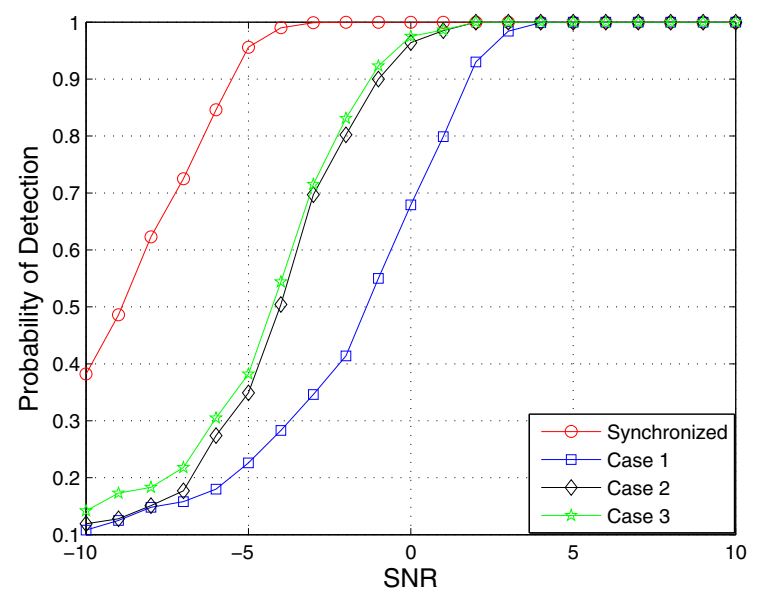

Fig. 3. Probability of detection vs. SNR for four-tap channel.
The probability of detection for all the cases is plotted for four and eight tap channel in Fig. 3 and 4 respectively. Both Figures are generated for SNR values $-10 \mathrm{~dB}$ to $10 \mathrm{~dB}$ at $P_{f}=0.1$ and $k=10$ is used for case 3 . In both figures, we compare the performance with synchronized energy detector according to equations from [4] where decision statistics and

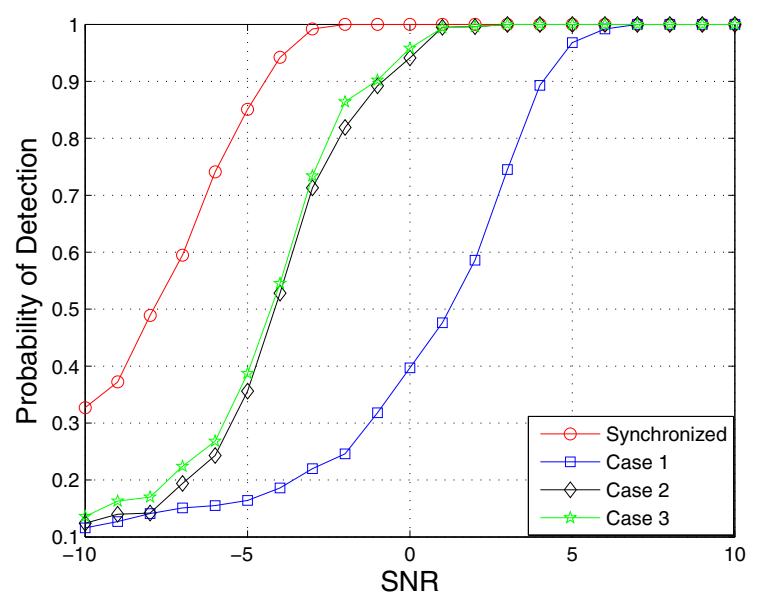

Fig. 4. Probability of detection vs. SNR for eight taps channel.

probability of false alarm are given by

$$
D_{e d} \cong \frac{2}{\sigma_{n}^{2}} \sum_{i=0}^{N-1}\left|r_{i}\right|^{2} ; \quad P_{f, e d}=1-P\left(\frac{V_{T}}{2}, N\right) .
$$

Note that $P(a, b)$ is the lower incomplete gamma function. Since the synchronized energy detector knows exact location of the primary signal and only examines two OFDM symbol period containing the primary signal, its performance is always high. From Fig. 3, it can be verified that without synchronization, our proposed system can achieve about $90 \%$ or above $P_{d}$ at SNR value as low as $-1 \mathrm{~dB}$ for case 2 and 3 and $2 \mathrm{~dB}$ for case 1. For eight-tap channel in Fig. 4, the same performance can be achieved for case 1 at SNR value as low as $4 \mathrm{~dB}$.

\section{REFERENCES}

[1] A. Ghosh, J. Zhang, J. G. Andrews and R. Muhamed, Fundamentals of LTE. Prentice Hall, 2010.

[2] S. Sesia, I. Toufik and M. Baker, LTE the UMTS long term evolutionfrom theory to practice. J. Wiley and Sons Ltd., 2009.

[3] M. M. Sebdani and M. J. Omidi, "Detection of an LTE signal based on constant false alarm rate methods and Constant Amplitude Zero Autocorrelation sequence," International Conference on Intelligent and Advanced Systems (ICIAS), pp. 1-6, Jun. 2010.

[4] M. Naraghi-Pour and T. Ikuma, "Autocorrelation-based spectrum sensing for cognitive redios," IEEE Transactions on Vehicular Technology, vol. 59, no. 2, pp. 718-733, Feb. 2010.

[5] J. Kim, J. Han, H. Roh and H. Choi, "SSS detection method for initial cell search in 3GPP LTE FDD/TDD dual mode receiver," 9th International Symposium on Communications and Information Technology, ISCIT 2009.

[6] 3GPP TS 36.942 v10.3.0, "Physical Channel and Modulation," Mar. 2011. 